\title{
HD 95881: a gas rich to gas poor transition disk?^
}

\author{
A. P. Verhoeff ${ }^{1}$, M. $\mathrm{Min}^{2}$, B. Acke ${ }^{3, \star \star}$, R. van Boekel $^{4}$, E. Pantin ${ }^{5}$, L. B. F. M. Waters ${ }^{1,3}$, A. G. G. M. Tielens ${ }^{6}$, \\ M. E. van den Ancker ${ }^{7}$, G. D. Mulders ${ }^{1,8}$, A. de Koter ${ }^{1,2}$, and J. Bouwman ${ }^{4}$ \\ 1 Astronomical Institute "Anton Pannekoek", University of Amsterdam, PO Box 94249, 1090 GE Amsterdam, The Netherlands \\ e-mail: verhoeff@uva.nl \\ 2 Astronomical Institute Utrecht, University of Utrecht, PO Box 80000, 3508 TA Utrecht, The Netherlands \\ Institute for Astronomy, KU Leuven Celestijnenlaan 200B, 3001 Leuven, Belgium \\ ${ }^{4}$ Max Planck Institut für Astronomie, Königstuhl 17, 69117 Heidelberg, Germany \\ 5 CEA/DSM/DAPNIA/Service d'Astrophysique, CE Saclay, 91191 Gif-sur-Yvette, France \\ ${ }^{6}$ Leiden Observatory, Niels Bohrweg 2, 2300 RA Leiden, The Netherlands \\ 7 European Southern Observatory, Karl-Schwarzschild-Strasse 2, 85748 Garching bei München, Germany \\ 8 SRON Netherlands Institute for Space Research, PO Box 800, 9700 AV Groningen, The Netherlands
}

Received 8 June 2009 / Accepted 30 March 2010

\section{ABSTRACT}

\begin{abstract}
Context. Based on the far infrared excess the Herbig class of stars is divided into a group with flaring circumstellar disks (group I) and a group with flat circumstellar disks (group II). Dust sedimentation is generally proposed as an evolution mechanism to transform flaring disks into flat disks. Theory predicts that during this process the disks preserve their gas content, however observations of group II Herbig Ae stars demonstrate a lack of gas.

Aims. We map the spatial distribution of the gas and dust around the group II Herbig Ae star HD 95881.

Methods. We analyze optical photometry, $Q$-band imaging, infrared spectroscopy, and $K$ and $N$-band interferometric spectroscopy. We use a Monte Carlo radiative transfer code to create a model for the density and temperature structure which quite accurately reproduces all the observables.

Results. We derive a consistent picture in which the disk consists of a thick puffed up inner rim and an outer region which has a flaring gas surface and is relatively void of "visible" dust grains.

Conclusions. HD 95881 is in a transition phase from a gas rich flaring disk to a gas poor self-shadowed disk.
\end{abstract}

Key words. infrared: stars - stars: pre-main sequence - stars: planetary systems: protoplanetary disks

\section{Introduction}

Herbig Ae (HAe) stars are known to have gas-rich, dusty disks that are the remnant of the star formation process. These disks are most likely the sites of ongoing planet formation. The processes leading to and associated with planet formation modify both the composition and the geometry of the disk. Grain growth and grain settling are expected to result in large spatial variation of the grain size distribution and the gas to dust mass ratio within the disk. The gravitational interaction of proto-planets with the disk can create gaps/holes. Also the gas and the dust chemistry is expected to vary spatially. In order to understand planet formation, it is thus important to establish the spatial distribution of gas and dust in protoplanetary disks independently.

Observationally, the spectral energy distributions (SEDs) of HAe stars have been divided into two groups, that reflect differences in the slope of the mid-IR $(10-60 \mu \mathrm{m})$ spectral range (Meeus et al. 2001). Group I sources have red SEDs, while group II sources have blue SEDs. These differences can be interpreted in terms of the geometry of the disk. The direct irradiation of the inner rim of a disk with an inner hole causes it to be puffed up

* Based on observations collected at the European Southern Observatory, Chile. Under program IDs: 060.A9234A, 076.C-0159A, 077.C-0367A, 078.C-0281.

$\star \star$ Postdoctoral Fellow of the Fund for Scientific Research, Flanders.
(Dullemond et al. 2001). This puffed up inner rim casts a shadow, and only the outer disk surface regions emerge from the shadow and receive direct stellar light. Depending on the dust opacity, some disks may never emerge from the shadow of the inner rim (Dullemond \& Dominik 2004). This provides an elegant explanation for the observed two types of SEDs: group I sources being flaring, and group II sources self-shadowed. This interpretation has been confirmed using spatially resolved mid-infrared (IR) imaging with the Very Large Telescope Interferometer (VLTI; e.g. Leinert et al. 2004).

A difference between group I and group II sources was also found for the strength of the mid-IR emission bands attributed to polycyclic aromatic hydrocarbons (PAHs; Meeus et al. 2001; Acke \& van den Ancker 2004): flaring disks tend to show strong PAH emission while self-shadowed sources show weaker or no PAH emission (Acke et al. 2010). A similar difference was found for the strength of the [OI] $6300 \AA$ line (Acke et al. 2005). However, there is significant scatter in these trends (see below). Both the PAHs and the [OI] line strength probe the gas in the upper disk layers, and both require direct irradiation of the disk surface by stellar photons to be excited. PAHs mainly probe the disk on scales of several tens to $100 \mathrm{AU}$ (e.g. van Boekel et al. 2004; Lagage et al. 2006; Geers et al. 2007; Verhoeff et al. 2010), i.e. similar scales as the dust continuum emission in the $10-60 \mu \mathrm{m}$ wavelength range. These observations suggest that the spatial 
distribution of gas in group II sources is different from that of group I sources: apparently, in group II sources the gas in the surface of the outer disk does not receive direct stellar photons.

In a theoretical study, Dullemond et al. (2007) show that for disks in which the dust settles but the scale-height of the gas does not change, both group I and group II sources should show prominent PAH emission, contrary to the observed trend. However, some disks classified as group II sources (i.e. with a self-shadowed dust geometry) are observed to show prominent PAH emission and [O I] line emission; examples are HD 98922 and HD 95881 (Acke et al. 2005; Acke \& van den Ancker 2004). Verhoeff et al. (2010) show that for HD 95881 the PAH emission is extended at a scale similar to those of group I sources. Fedele et al. (2008) and van der Plas et al. (2008) studied three $\mathrm{HAeBe}$ stars and found that the gas and dust in their disks has a different spatial distribution. In the case of the group II source HD 101412, PAH and [O I] emission were detected and found to be more extended than the dust continuum at $10 \mu \mathrm{m}$. These observations suggest that disks exist in which the dust has settled but the scale-height of the gas is still large enough at several tens of $A U$ distance from the star to produce substantial PAH and [OI] line emission. Such disks may provide important clues as to how gas-rich disks evolve to gas-poor debris disks.

In this paper, we study the spatial distribution of the gas and dust in the disk of HD 95881. This star was part of a larger study of spatially resolved mid-IR spectroscopy of HAe stars (Verhoeff et al. 2010). We use optical spectroscopy of the [OI] line, the SED, infrared spectra as well as near-IR and mid-IR interferometric observations to constrain the geometry of the gas and dust in the disk. We use a hydrostatic equilibrium disk model to fit the SED and compare the predicted spatial distribution of the near-IR and mid-IR emission of the best fitting disk model to the interferometric observations. We find convincing evidence that the dust in the disk of HD 95881 has settled but that the gas still has a significant scale-height. We derive an estimate on the total disk mass by fitting the strength of the PAH bands.

\section{Stellar parameters}

There is little information available on HD 95881 in the literature. From optical photometry the spectral type was determined to be A2III/IVe (Houk \& Cowley 1975), which was translated into an effective temperature of $8990 \mathrm{~K}$ (Acke \& van den Ancker 2004). The distance was established by a relatively uncertain association with the star formation region Sco OB2-4 (Acke \& van den Ancker 2004). The distance to this region was previously determined by de Zeeuw et al. (1999). When we take these parameters to pinpoint HD 95881 in the Hertzsprung-Russelldiagram, we find that it is situated to the left of the Zero Age Main Sequence (ZAMS), which is unphysical. Since we consider the determination of $T_{\text {eff }}$ as reliable, we adopted a luminosity of $15.4 L_{\odot}$ assuming the ZAMS luminosity from Meynet et al. (1994) at the given $T_{\text {eff }}$. The corresponding ZAMS mass was adopted as the stellar mass. From the new luminosity, new estimates for the radius and distance follow. Table 1 lists the basic characteristics and our new estimates. To double check the new distance we looked at the Tycho parallax, which gave a lower limit of $80 \mathrm{pc}$. We also consulted the Hipparcos cata$\log$ for spectral types and $(B-V)$ photometry of stars within $1^{\circ}$ from HD 95881 and compared the distance to the color excess $E(B-V)$ for the region (de Zeeuw et al. 1999). For HD 95881 the color excess of $E(B-V)=0.08$ leads to an upper limit on the distance of $\sim 200 \mathrm{pc}$. The derived distance interval of 80-200 pc is consistent with both distance estimates, but because of the
Table 1. Characterizing parameters of HD 95881.

\begin{tabular}{lcc}
\hline \hline Parameter & Value & New \\
\hline Right Ascension & $11^{\mathrm{h}} 01^{\mathrm{m}} 57^{\mathrm{s}} .62$ & \\
Declination & $-72^{\circ} 30^{\prime} 48^{\prime \prime} 4$ & \\
Spectral Type & A2III/IVe & \\
$T_{\text {eff }}[\mathrm{K}]$ & 8990 & \\
Distance [pc] & $118 \pm 12$ & $170 \pm 30$ \\
Luminosity $\left[L_{\odot}\right]$ & $6.9 \pm 1.0$ & $15.4 \pm 6$ \\
$A_{\mathrm{V}}[\mathrm{mag}]$ & 0.25 & \\
Radius $\left[R_{\odot}\right]$ & $1.1 \pm 0.1$ & $1.6 \pm 0.3$ \\
Mass $\left[M_{\odot}\right]$ & - & $2.0 \pm 0.3$ \\
SFR & Sco OB2-4? & $?$ \\
Group & IIa & \\
\hline
\end{tabular}

Notes. The second column lists parameters as given by Acke \& van den Ancker (2004), where SFR stands for the star formation region. New values for some of the parameters are given in the third column.

argument given above we adopt a distance estimate as derived from the ZAMS of $170 \pm 30 \mathrm{pc}$.

\section{Observations}

\section{1. $A M B E R$}

Spectrally dispersed $K$-band interferometric observations of HD 95881 were obtained with VLTI/AMBER on the UT1-UT3UT4 baseline setting during the night of February 4, 2007. The weather conditions were excellent, with the optical seeing as low as $0.5^{\prime \prime}$. The data have been reduced according to the standard AMBER data reduction procedure described in Tatulli et al. (2007). AMBER observations of a standard star (HD 100901, K0/K1III) were performed directly before the science measurement and used to calibrate the raw visibilities.

\subsection{VISIR imaging}

$Q$-band imaging data were obtained on the 14 th of December 2005 using the VLT Imager and Spectrometer for mid-Infrared (VISIR; see Lagage et al. 2004). Standard "chopping and nodding" mid-infrared observational technique was used to suppress the background dominating at these wavelength. The $Q 2$ filter was chosen, which has a central wavelength of $18.72 \mu \mathrm{m}$ and a half-band width of $0.88 \mu \mathrm{m}$. The pixel field of view was $0.075^{\prime \prime}$ and the orientation was standard (north up, east to the left). The total integration time spend on HD 95881 was $680 \mathrm{~s}$. The reference star, HD 1024601 (10.6 Jy in the $Q 2$ filter), was chosen from the database of VISIR standard stars based on criteria of flux and distance on the sky to the target. $456 \mathrm{~s}$ of integration time was spent on HD 102461. The airmass of both sources was always below 1.5. The sensitivity achieved was estimated to be $57 \mathrm{mJy} / 10 \sigma / 1 \mathrm{~h}$. The optical seeing was moderately fluctuating in the range $0.75-0.85^{\prime \prime}$, corresponding to an estimated $Q$-band seeing of $\lesssim 0.4^{\prime \prime}$.

\subsection{VISIR spectroscopy}

Long slit $N$-band spectra were obtained with VISIR in the low resolution (LR) mode. A sample of $17 \mathrm{HAe}$ stars was observed under the VISIR GTO program on circumstellar disks (Verhoeff et al. 2010). During the nights of December 16, 2005 and April 8, 2006 we observed HD 95881. Standard chopping and nodding along the slit was used with a chopper throw of $8^{\prime \prime}$, a slit-width of 
$0.75^{\prime \prime}$, and a standard North-South orientation. After and before the science measurements bright standard stars were observed. The airmass of the observations was $\sim 1.6$ and the optical seeing was around 1.0" during the first night and around 1.2" during the second night, corresponding to an estimated $N$-band seeing of $\lesssim 0.65^{\prime \prime}$.

\section{4. $\mathrm{MIDI}$}

HD 95881 was observed with the MID-infrared Interferometric instrument (MIDI; see Leinert et al. 2003), the $10 \mu \mathrm{m}$ instrument of the VLTI, during the night starting June 6, 2004, as part of the "science demonstration" program. The UT1-UT3 baseline was used, resulting in a projected baseline length of $65 \mathrm{~m}$ at a position angle of $81^{\circ}$ east of north. The grism was used to spectrally disperse the signal at a resolution of $R=\lambda / \Delta \lambda=230$. This ensures that relatively narrow "dusty" emission features such as those of crystalline silicates and PAHs are well resolved spectrally. The seeing was constant at a value of $0.6^{\prime \prime}$ and the atmospheric transparency was excellent.

We performed observations in "High-Sens" mode, i.e. we took an interferometric measurement combining the light from both telescopes, and subsequent photometric measurements of the signal from one telescope at a time. Stars of known brightness and angular diameter were observed, using the same procedure, for photometric calibration and monitoring system coherence losses (interferometric "transfer function"). We found our interferometric measurement of HD 95881 to be of significantly higher quality than the corresponding photometric measurements, and chose to directly calibrate the correlated flux rather than converting to interferometric visibility by division over the photometry.

\subsection{Additional data}

A low resolution Spitzer-IRS spectrum is used to compare and flux-calibrate the VISIR spectrum. For the acquisition and reduction of the Spitzer data we refer to Juhász et al. (2010). Photometric data points were taken from Acke \& van den Ancker (2004). Together with the Spitzer spectrum they make up the SED, which allows us to constrain the disk geometry (see Sect. 5). Finally, [O I] data were taken from an earlier study, see Acke et al. (2005).

\section{Analysis}

\section{1. $A M B E R$}

A good fit $\left(\chi^{2} / v=1.8\right)$ to the AMBER data could be obtained with an inclined uniformly emitting ring surrounding a pointlike source representing the star (Fig. 1). The inner and outer radius of this ring are $2.2 \pm 0.2$ mas $(0.37 \mathrm{AU})$ and $2.7 \pm 0.3$ mas ( $0.46 \mathrm{AU})$. The disk inclination is $60^{\circ} \pm 10^{\circ}$ and its position angle is $102^{\circ} \pm 2^{\circ}$. The mean radius of the ring is 2.4 mas $(\sim 0.4 \mathrm{AU})$. This is similar to the values found by Eisner et al. (2004) for a group of Herbig Ae/Be stars. The fraction of the $K$-band flux that is ascribed to the star by the fit of a Kurucz model to the optical photometry $(\sim 20 \%)$ is not exactly equal to the AMBER estimate of $32 \pm 4 \%$. It may be that this discrepancy is due to the presence of an unresolved disk component, e.g. hot gas well within the dust sublimation radius, that contributes to the $K$-band emission. Gas emission from a very compact region around the star, where no dust species can survive, has been claimed for quite a number of pre-main-sequence stars (Eisner et al. 2007;

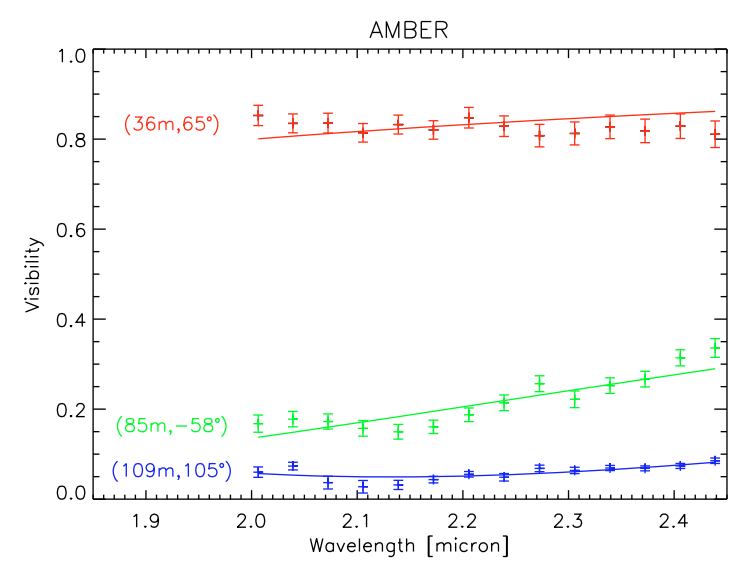

Fig. 1. The AMBER $K$-band visibilities of HD95881. The projected length and position angle of the baselines is indicated on the left. Overplotted is the best-fit ring+point-source model (solid lines; see text).

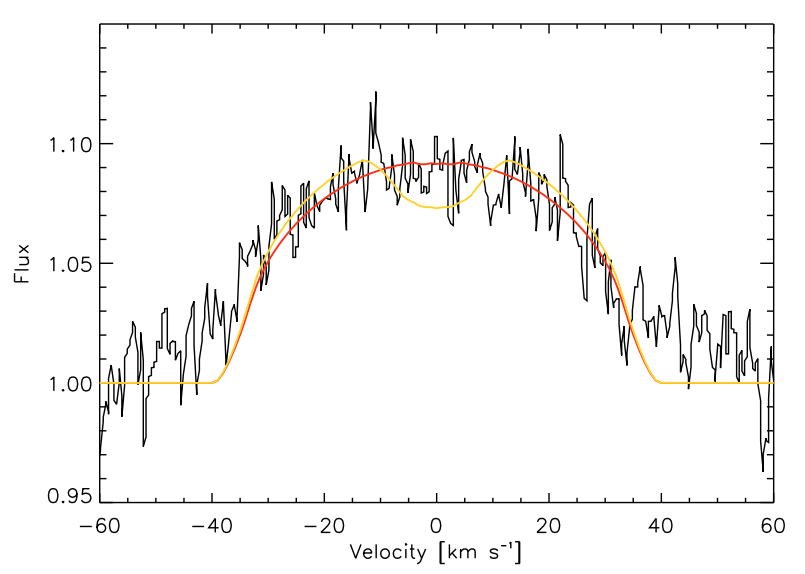

Fig. 2. The continuum-normalized [OI] $6300 \AA$ emission line of HD 95881 (Acke et al. 2005). The velocity axis has been centered on the centroid position of the feature, i.e. the radial velocity of the central star. Line profiles corresponding to a surface brightness proportional to $r^{-3}$ are overplotted. Red represents a model with an outer radius beyond $31 \mathrm{AU}$; yellow corresponds to the model with an outer radius equal to 11 AU. See text for details.

Kraus et al. 2008; Acke et al. 2008; Tannirkulam et al. 2008b; and Isella et al. 2008). However, refractory dust has also been suggested to be present in the innermost regions (Benisty et al. 2009).

\section{2. [OI] data}

Acke et al. (2005) have investigated the [O I] $6300 \AA$ emission line in a large sample of Herbig stars. The authors argue that the emission is non-thermal and originates from the disk surface of a flared circumstellar disk. This is consistent with the large detection rate of [O I] emitters among the group I sources in the sample. Roughly half of the group II sources, however, display the [O I] $6300 \AA$ line in emission as well, albeit less strong. HD 95881 is one of these targets.

In Fig. 2, the [O I] $6300 \AA$ line profile is shown. We have fitted a generic model to the data, assuming that the intensity drops off with radius as a power law and that the disk is in Keplerian rotation. We adopt the disk inclination derived from the AMBER fit. An acceptable fit (reduced $\chi^{2}=3$ ) was achieved with a power 


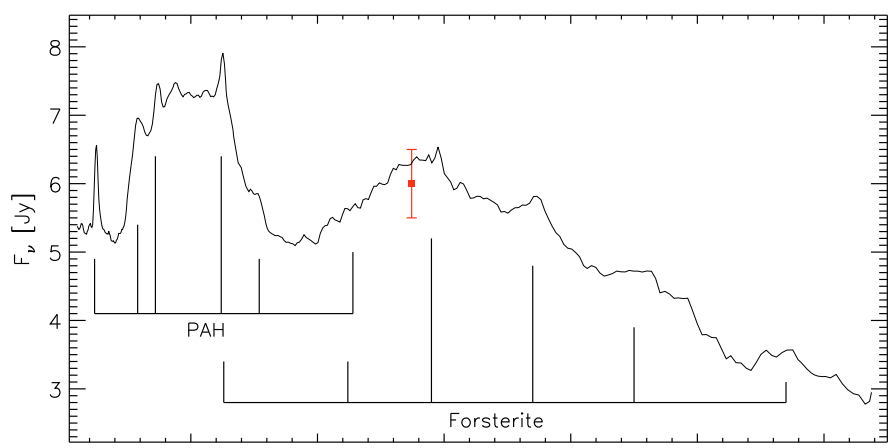

Fig. 3. The Spitzer LR spectrum of HD $95881(S N R \approx 500)$. The left rake demonstrates the presence of the PAH bands at 6.2, 7.9, 8.6, 11.2, 12.7 , and $16.4 \mu \mathrm{m}$. The right rake points at the forsterite bands at 11.3, $16.2,19.5,23.5$, and $33.5 \mu \mathrm{m}$. The red square is the photometry from the VISIR $Q$-band imaging (see Sect. 4.4).

index of $-3.0 \pm 0.2$, an inner radius of $0.9 \pm 0.2 \mathrm{AU}$ and an outer radius larger than $11 \mathrm{AU}$. The best fit (reduced $\chi^{2}=1.5$ ) has an outer radius beyond $31 \mathrm{AU}$. In the figure we show the bestfit profile, as well as the profile that corresponds to the model with an $11 \mathrm{AU}$ outer radius. Decreasing the outer radius of the model to even lower values would further deteriorate the fit quality. We can therefore conclude that a significant fraction of the [O I] emission comes from a region at tens of $\mathrm{AU}$ from the star. Note that our model does not account for the $15 \%$ of [O I] flux which is emitted in the blue- and red wings of the profile. In these regions, close to the star, the power law approximation fails.

\subsection{Spitzer}

The Spitzer-IRS spectrum is given in Fig. 3. It displays a rich mineralogy, there is emission of amorphous and crystalline silicates as well as emission of various PAH bands. See Juhász et al. (2010) for a detailed discussion of the mineralogy. The blue slope at longer wavelengths is typical for group II sources.

Compared to other HAe stars HD 95881 appears to be a very ordinary PAH emitter. We have verified this by comparing PAH band-strength ratios. We have taken the 6.2, 7.7, 8.6, 11.2 and $12.7 \mu \mathrm{m}$ PAH band strengths from Verhoeff et al. (2010) and Acke et al. (2010) and looked at the ratios of their continuum subtracted and integrated strengths. The only aspect that makes HD 95881 stand out slightly is the $8.6 \mu \mathrm{m}$ PAH feature, which is relatively strong.

\subsection{VISIR imaging}

A dedicated data reduction pipeline was used for the imaging. It features a comprehensive set of methods to correct for instrumental signatures such as detector striping or background low-frequencies excess of noise (Pantin et al. 2008, 2009). A photometric analysis gives an integrated flux for HD 95881 of $6.0 \pm 0.5 \mathrm{Jy}$ in the $Q 2$ filter $(18.72 \mu \mathrm{m})$. This is in fair agreement with the Spitzer data (see Fig. 3). Since the emission of the star is negligible at this wavelength, this flux can be attributed to the disk. We searched for an extended emission component using PSF subtraction at a sub-pixel (1/10) precision level. The PSF was derived from the observation of the standard star. The resulting residuals display an excess of signal that is roughly axi-symmetric and decreases gradually as a function of distance from the star.

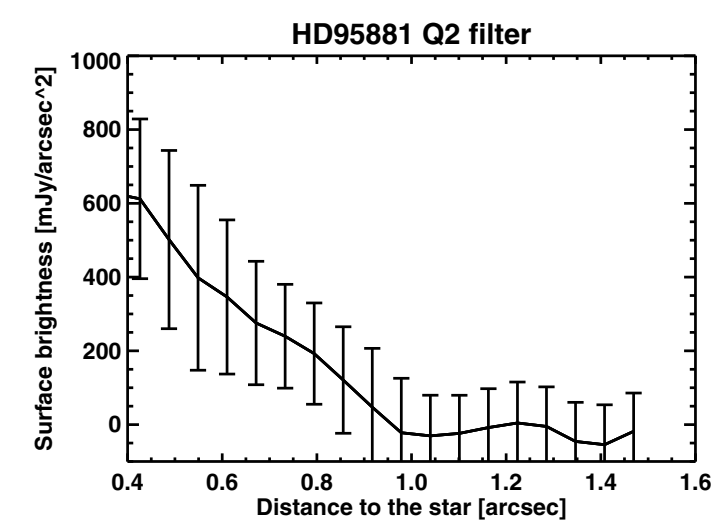

Fig. 4. Surface brightness levels as a function of distance from the star in the $Q 2$ image after point source subtraction. The error bars give the $3 \sigma$ uncertainty.

Assuming that the disk has the geometric parameters given in Table 2, we divided the residuals in a series of concentric ellipses having a separation of $0.075^{\prime \prime}$ along the semi-major axis of the disk. We assumed an aspect ratio corresponding to a flat disk inclined at $55^{\circ}$, and a position angle of $103^{\circ}$ from North. We used these elliptic annuli to numerically estimate the surface brightness distribution and the corresponding uncertainty levels.

Our statistical analysis confirms we have a significant detection since for distances in the range $0.4-0.85^{\prime \prime}$ from the star the average values in each of the elliptical annuli are well above the detection limits set at $99 \%$ confidence. The measured average surface brightness in elliptic annuli as a function of the semimajor axis distance to the star are plotted in Fig. 4. The $3 \sigma$ uncertainties are displayed as bars on the plot. The total flux in the resolved component is $0.7 \pm 0.1 \mathrm{Jy}$.

\subsection{VISIR spectroscopy}

The general reduction and analysis strategy of the spectroscopic VISIR data is described in detail in Verhoeff et al. (2010). For HD 95881 the telluric correction was done by means of an airmass interpolation of two calibrators. The observation of April 8, 2006, appeared to be suffering strong atmospheric residuals. The spectrum of December 162005 was thus chosen as most representative. The missing observation of the $9.8 \mu \mathrm{m}$ setting was replaced with the poor one from April 8, 2006. We scaled the VISIR spectrum to the Spitzer spectrum at $10.6 \mu \mathrm{m}$ using an average factor for the different settings of 1.16. The resulting spectrum in Fig. 5 has a SNR of $\sim 300$. The agreement with the Spitzer spectrum is encouraging. The slight deviation observable just left of the ozone band at $9.6 \mu \mathrm{m}$ is typical for the quality of the data taken on April 8, 2006.

The Full Width at Half Maximum (FWHM) of the spatial emission profile of the target was determined by performing a Gauss-fit in 32 merged wavelength bins. Comparison of the science signals with the PSF shows that the target is unresolved in the continuum. After quadratic subtraction of the PSF and averaging over the median values of all measurements we find a three sigma upper-limit to the FWHM extent of the continuum emission region of $<0.46^{\prime \prime}$, which corresponds to $<79 \mathrm{AU}$ at the adopted distance of $170 \mathrm{pc}$. However the science signal displays a relative increase at 8.6 and $11.2 \mu \mathrm{m}$ and an upturn to the left of $\sim 8 \mu \mathrm{m}$, which are exactly the wavelengths at which the PAH molecules have emission features (see Verhoeff et al. 2010). We checked the significance of these $F W H M$ features with respect 


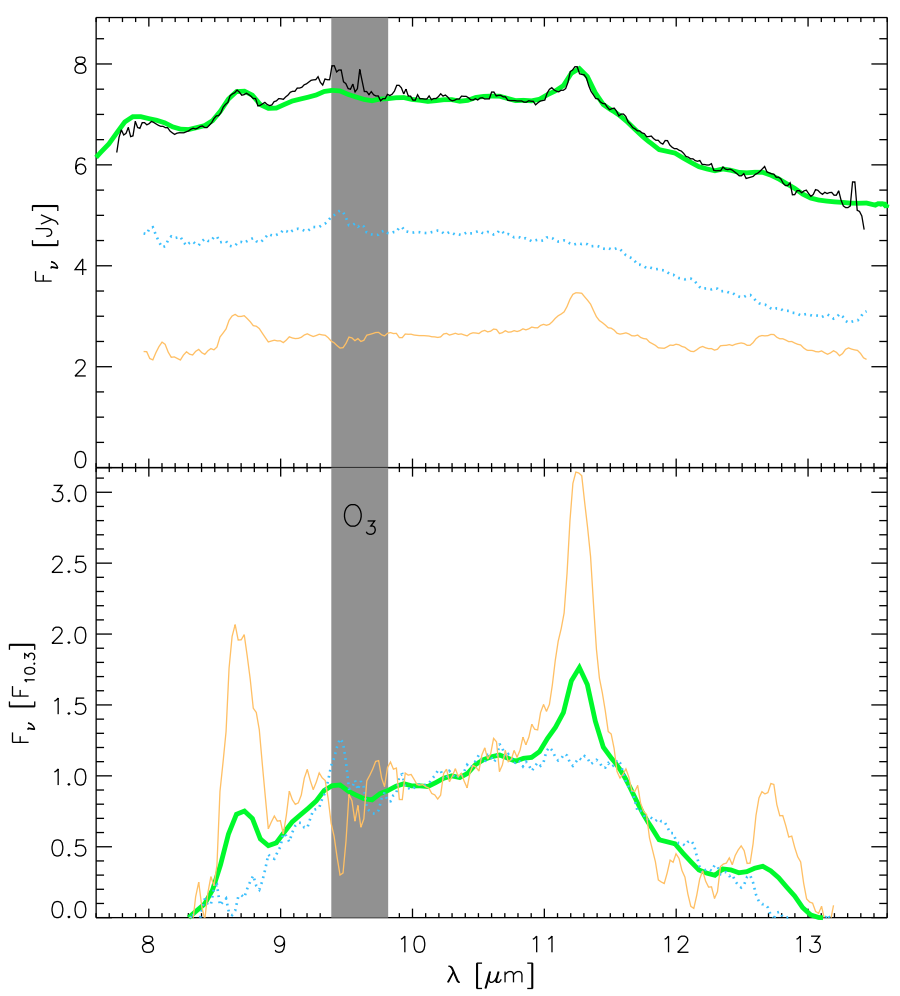

Fig. 5. The top panel shows the VISIR N-band spectrum of HD 95881 (black). Overplotted are the Spitzer spectrum (thick green), the correlated flux as measured with MIDI (dotted blue), and the difference between the Spitzer and MIDI spectra (orange). The Spitzer spectrum represents the entire disk, the MIDI correlated flux spectrum represents the inner disk ( $r \lesssim 2 \mathrm{AU})$, and the difference spectrum represents the outer disk $(2 \lesssim r \lesssim 100 \mathrm{AU})$. The bottom panel shows the normalized continuum subtracted silicate feature (same color coding). The similar shape of the inner and outer disk silicate feature indicates a similar composition and grain size distribution.

to pixel-to-pixel variations and concluded that the PAH emission is significantly more extended than the continuum.

In order to estimate the spatial extent of the PAH emission we measured the spatial emission profile at the peak wavelengths of the PAH bands and we subtracted the spatial emission profile of the continuum contribution. This continuum profile was determined by interpolating the intensities and spatial profiles adjacent to the PAH bands. The resulting observed spatial profile of the PAH emission was Gaussian fitted to obtain the FWHM. Finally, the instrumental width (i.e. the PSF) was quadratically subtracted to obtain a measure for the intrinsic extent of the PAH emission. We found $F W H M$ values of $0.34^{\prime \prime+0.05}$ and $0.39^{\prime \prime}{ }_{-0.06}^{+0.04}$ for the 8.6 and $11.2 \mu \mathrm{m}$ PAH bands respectively, which results in absolute sizes of 58 and 66 AU. In a Gaussian distribution of the PAH emission this would mean that $99 \%$ is confined in a radius of $\sim 100 \mathrm{AU}$. Note that this is a conservative estimate of the PAH emission scale since the PAH surface brightness is expected to fall off with distance from the star together with the stellar flux as $1 / r^{2}$.

\subsection{MIDI}

In Fig. 5 we compare the spectrum in correlated flux as seen by MIDI, i.e. the total flux times the visibility, to the spectra observed by VISIR and Spitzer. The correlated flux spectrum is dominated by the central few AU of the disk, the Spitzer and VISIR spectra probe the entire disk. Note the difference in the strength of the 8.6 and $11.2 \mu \mathrm{m}$ PAH bands in the spectra. These bands are prominent in the total flux spectra, but essentially absent in the correlated flux spectrum. The PAH emission region is apparently outside of the disk region probed by MIDI. This shows by direct measurement that the PAH features arise at scales much larger than $2 A U$ in the disk of HD 95881. To stress this point we also plotted the difference between the Spitzer and the MIDI correlated flux spectrum in the bottom panel of Fig. 5. This difference spectrum is dominated by the emission of the outer disk ( $2 \lesssim r \lesssim 100 \mathrm{AU})$ and shows very distinct PAH features.

To investigate spatial differences in the weak silicate emission we considered the shape of the feature in the Spitzer, MIDI, and difference spectrum in a consistent manner. We approximated a continuum with a straight line intersecting the spectra at 8.3 and $13.2 \mu \mathrm{m}$, subtracted this from the spectra and then normalized the spectra with the flux level at $10.3 \mu \mathrm{m}$. The bottom panel of Fig. 5 shows the result. The shape of the silicate feature inside and outside a radius of $\sim 2$ AU is very similar, which implies that the silicate composition and grain size distribution should also be very similar. This is not surprising, since the high visibility indicates that the silicate emission is dominated by a compact inner region $(r \lesssim 10 \mathrm{AU})$.

\subsection{Observational picture}

Before we describe a detailed modeling effort of the circumstellar material, we summarize the analyses of the various observations. This already gives an insight into the spatial distribution of the gas and the dust. In Fig. 6 we display schematic representations of two disk models, that will be considered in Sect. 5. In the second model we indicated the diagnostic scales of the various data sets to combine them into a consistent picture.

The AMBER data probes the very inner parts of the disk. The analysis shows that the $K$-band emission could be explained with an emitting ring at $\sim 0.4 \mathrm{AU}$. A more physical model, which will be presented in Sect. 5 has the inner rim of the disk at $0.55 \mathrm{AU}$. A part of the $K$-band emission was shown to come from close to the star, and well within the inner dust disk (represented by the ring). This could be an indicator of ongoing accretion.

The [OI] $6300 \AA$ emission line is formed by the photodissociation of $\mathrm{OH}$ molecules by UV photons (Acke et al. 2005). The detection of the [O I] $6300 \AA$ at large distances (from one to tens of AU) from the central star is thus an indication that the outer disk has an illuminated gas surface.

The Spitzer data establish the presence of PAH emission and the VISIR spectrum pins it down to a circumstellar disk. The emission features of PAH molecules are caused by internal vibrational modes, which are mainly excited by UV photons. Since the PAH molecules are coupled to the gas, the PAH emission is another indicator of an illuminated gas surface. The resolved VISIR spectrum sets the radial scale of this gas surface at $\sim 100 \mathrm{AU}$.

The VISIR $Q$-band image displays a faint extended axisymmetric emission component ( $\sim 10 \%$ of the total flux), that stretches out to large radii $(r \sim 150 \mathrm{AU})$.

The MIDI correlated flux spectrum shows that the PAH features originate from radii much larger than $\sim 2 \mathrm{AU}$ and that the silicate composition is quite similar in the inner and outer disk. 


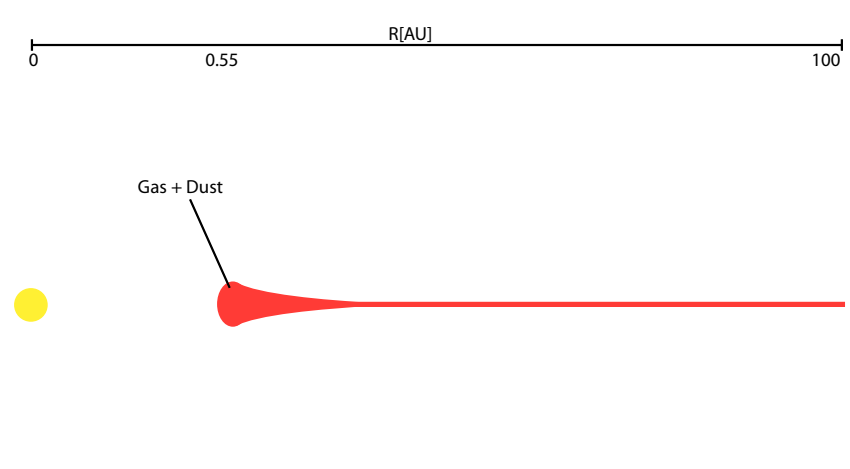

Model 1

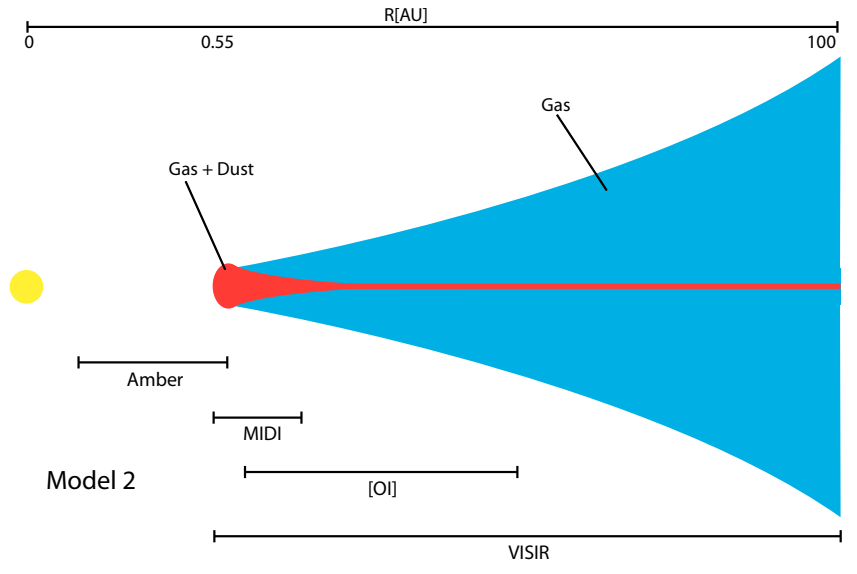

Fig. 6. Schematic depiction of the two considered disk models. First we consider a spatial distribution that is the same for both the dust and the gas. Second we consider a model that assumes that the surface density of the gas decays much slower with distance from the star. In this second model we indicated the different regions of the disk that are probed by the observations discussed in this paper.

\section{Modeling}

In this section we present a model for the disk around HD 95881 which quite accurately reproduces the observations described above. To obtain this model we use the Monte Carlo radiative transfer code MCMax by Min et al. (2009). This code can compute a self-consistent disk structure and a full range of observables. It has a build-in option that models the full PAH excitation, using the temperature distribution approximation (see e.g. Guhathakurta \& Draine 1989; Siebenmorgen et al. 1992) including multi-photon events for the excitation. We use MCMax here to compute the temperature structure, the vertical density structure and the resulting SED, the Spitzer spectrum, the AMBER visibilities, the MIDI correlated flux, the VISIR images, and the FWHM as function of wavelength. The steps to come to the final model presented here were the following.

\subsection{Initial constraints}

First we fixed the composition, and the size and shape distribution of the silicate component of the dust to be equal to that obtained from the 10 micron silicate feature by van Boekel et al. (2005) . For HD 95881 these are $80 \%$ large $(1.5 \mu \mathrm{m})$ pyroxene grains, $11 \%$ large enstatite grains, $1 \%$ small $(0.1 \mu \mathrm{m})$ forsterite grains, 5\% large forsterite grains, and 3\% small $(0.1 \mu \mathrm{m})$ silica grains. For the shapes of the grains we use the Distribution of Hollow Spheres (DHS, see Min et al. 2005), as outlined in van Boekel et al. (2005), where we refer to for further details on the model for the silicate dust grains. In order to get the required continuum opacity needed we added amorphous carbon grains. To model the irregular shape of the carbon grains we also used DHS. For the refractive index of carbon we adopted the data by Preibisch et al. (1993). Note that the continuum component is most likely not all in the form of amorphous carbon. Small grains of metallic iron and/or iron sulfide have extinction properties similar to that of carbon. Also, large grains of various dust species could produce the observed continuum component. The abundance of amorphous carbon is a fitting parameter. We have to realize that besides this observable dust component, a "hidden" component of large grains probably exists deep inside the disk. Information on this dust component, which might contain most of the dust mass, can only be obtained through millimeter observations which are currently not available for this source. In the following we will use the term "visible" for the dust grains we can address with the current set of observations.

The other free parameters all have to do with the geometry of the disk. As a first step we focused on the thermal dust grains, ignoring the PAH bands. The density distribution of the dust disk was parameterized using a radial surface density (Hughes et al. 2008)

$\Sigma(r) \propto r^{-p} \exp \left\{-\left(\frac{r}{R_{0}}\right)^{2-p}\right\}$,

for $R_{\text {in }}<r<R_{\text {out }}$. Here $R_{0}$ is the turnover point from where an exponential decay of the surface density sets in and $p$ sets the powerlaw in the inner region. We fixed this powerlaw to $p=1$, a commonly used value (see e.g. Dullemond et al. 2006). Using $p=1$ basically implies that we assume the viscosity of the disk to linearly increase with radius. For a discussion on the possible values of $p$ and their implications for the origin of turbulence and viscosity in the disk we refer to Isella et al. (2009). The vertical density structure was computed from hydrostatic equilibrium. However, since we found that this vertical structure cannot reproduce the SED, we included a scaling parameter $\Psi$ by which the scale-height of the entire disk is increased. For the density distribution we thus have the inner and outer radii $R_{\text {in }}$ and $R_{\text {out }}$, the turnover radius $R_{0}$, the total dust mass in visible grains $M_{\text {dust }}$, and the vertical density scaling parameter $\Psi$, as free parameters.

\subsection{Fitting the SED}

In order to get the observed peak over continuum ratio of the 10 and 20 micron silicate features observed in the Spitzer spectrum, we find that the mass in amorphous carbon grains has to be $25 \%$ of the total dust mass. Again, we stress that this component represents all possible sources of continuum opacity, including very large grains. Second, we constrained the parameters describing the density structure. We found that the vertical height of the disk needs to be significantly increased compared to hydrostatic equilibrium in order to obtain the large near IR excess (see also Acke et al. 2009, for a discussion on the inner rim height). The height of the disk was scaled with a factor $\Psi=2.75$. The exponential decay of the surface density sets in at $R_{0}=2.5 \mathrm{AU}$. To put the density structure in some perspective, at $\sim 7.5 \mathrm{AU}$ our prescription produces the same density as does a $1 / r^{2}$ density law, but beyond $10 \mathrm{AU}$ the surface density becomes negligible. 
The inner radius of the disk is at $0.55 \mathrm{AU}$, while the outer radius has no influence on the observationally constrained part of the SED as long as it is beyond $\sim 10 \mathrm{AU}$. We find a total mass in visible dust grains of $10^{-8} M_{\odot}$. Note that the total dust mass is probably much higher, because the mass in large dust grains is not constrained. To this dust distribution we added PAHs in an abundance needed to explain the features seen in the Spitzer spectrum. For the opacity of the PAHs we use those computed by Draine \& Li (2001) for molecules consisting of 100 carbon atoms.

\subsection{Spatial distribution of the gas}

We consider two possibilities for the spatial distribution of the gas, as traced by the PAHs (see Fig. 6). The first is to assume that the gas and the dust have the same spatial distribution in the disk. The second is to assume that the gas does not have the exponential decay of the surface density for radii larger than 2.5 AU, but that this disappearance of the dust at these radii is caused by grain growth and settling, which do not affect the gas.

The first model, which has the gas and dust in the same spatial distribution, results in a fairly large abundance of PAH molecules needed to explain the strength of the features seen. The PAHs are in this case fairly well shielded from the stellar radiation by the dust grains, and thus a large amount is needed. Furthermore, in order to explain the absence of PAH emission in the MIDI correlated flux, we find that we have to remove the PAHs from the inner $2 \mathrm{AU}$. The total mass in PAHs in this case is $3 \times 10^{-8} M_{\odot}$. This model does not reproduce the increase in FWHM at the wavelength of the PAH features that we found in the VISIR spectroscopic data (see Sect. 4.5).

The second model, which assumes the gas is distributed in a more extended flared disk (see Fig. 6) resulted in a very good overall fit of all the observables presented above. In this model we put the PAHs in a disk with a similar surface density as the dust grains but with $R_{0}=\infty$, i.e. the surface density remains a powerlaw $(p=1)$ for all radii. In this way we create a flaring outer gas disk, which is able to catch much of the radiation from the star. We find that the PAH emission is dominated entirely by the outer regions. If the PAHs would be destroyed according to the mechanism proposed by Dullemond et al. (2007) we find that the inner $25 \mathrm{AU}$ should be free of PAHs. However, even without destruction the contribution to the PAH emission from these inner regions is negligible. Thus, we cannot confirm whether or not $\mathrm{PAH}$ destruction takes place in this disk. Extrapolating the powerlaw surface density distribution of the PAHs to the inner edge (at $0.55 \mathrm{AU}$ ) we find that at $R_{\text {in }}$ the PAH abundance is $0.25 \%$ of the visible dust mass. The total PAH mass we find is $5 \times 10^{-9} M_{\odot}$, which is large compared to the mass in visible dust grains, because the PAH disk is so much larger. Including PAH destruction in the inner disk would only lower the total PAH mass by $12 \%$. Note, that the total dust mass, including the well shielded midplane grains, can be much higher than the mass in visible grains and the PAH mass. This could be confirmed by future millimeter observations.

\subsection{Final model}

The second model, that assumes that the PAHs do not have an exponential decay with radius, reproduces the available spatial information much better. This second model was fine-tuned to best reproduce all available observables. The position angle and inclination of the disk were constrained using the interferometric
Table 2. The parameters for our final model for the circumstellar disk of HD 95881.

\begin{tabular}{lcc}
\hline \hline Parameter & Dust & PAHs \\
\hline Inner disk radius $\left(R_{\text {in }}\right)$ & $0.55 \mathrm{AU}$ & $0.55 \mathrm{AU}$ \\
Outer disk radius $\left(R_{\text {out }}\right)$ & $200 \mathrm{AU}$ & $200 \mathrm{AU}$ \\
Inclination angle $(i)$ & $55^{\circ}$ & $55^{\circ}$ \\
Position angle $($ major axis E of $\mathrm{N})$ & $103^{\circ}$ & $103^{\circ}$ \\
Vertical density scaling parameter $(\Psi)$ & 2.75 & 2.75 \\
Power law for the surface density $(p)$ & 1 & 1 \\
Turnover point $\left(R_{0}\right)$ & $2.5 \mathrm{AU}$ & $\infty$ \\
Mass $(M)$ & $10^{-8} M_{\odot}$ & $5 \times 10^{-9} M_{\odot}$ \\
\hline
\end{tabular}

Notes. The carbon abundance was found to be $25 \%$ of the visible dust. The visible dust grains and the PAH molecules have a different spatial distribution.

data. We find that the AMBER interferometric observations put important constraints on the parameters for the inner regions of the disk, lifting some of the degeneracies present when these data are not considered. The parameters of the final model are summarized in Table 2.

\section{Discussion}

\subsection{Comparison of the model with the observations}

Our disk model accurately reproduces all the observables. The fits to the SED, the Spitzer spectrum, the MIDI correlated flux, the AMBER visibilities, and the VISIR spectroscopic FWHM are presented in Fig. 7.

Spitzer. The general slope and most features in the Spitzer spectrum are reproduced. However, the observed 6.2, 7.9 and $8.6 \mu \mathrm{m}$ PAH features are stronger than in our model and the predicted PAH features around $20 \mu \mathrm{m}$ are not seen in the Spitzer spectrum. These differences are related to the PAH chemistry, which is a much debated subject (see e.g. Draine \& Li 2007).

MIDI. The $N$-band correlated flux obtained by MIDI has an error on the absolute calibration of approximately $10 \%$, similar to the difference with the model output. Thus our modeling of the spatial distribution of the visible dust grains is consistent with the MIDI result.

$A M B E R$. The simple flat ring+point-source model of Sect. 4.1 actually reproduced the inclination, position angle and visibilities of the disk very well, although the inner rim radius ( $0.37 \mathrm{AU}$ ) is significantly below the $0.55 \mathrm{AU}$ of our final model. Our more physical final model gives a slightly poorer fit to the visibilities. This indicates that the structure of the inner disk is more complicated than assumed. The exact structure is currently much debated (see Sect. 6.2).

VISIR spectrum. The model FWHM was obtained by making a Gaussian fit to the spatial profile after convolving the model with a Gaussian of the same width as the PSF of the VISIR $11.2 \mu \mathrm{m}$ setting. The PSF of the $8.5 \mu \mathrm{m}$ setting was scaled to the model. The matching continuum levels are thus a result of our method, but the increase in the FWHM at the PAH wavelengths are a confirmation of the correct modeling of the spatial distribution of the PAH emission.

VISIR image. A model of the $Q 2$ image was obtained by taking the output of our model at $19.0 \mu \mathrm{m}$, just next to the artificial PAH feature. We convolved this model image with the VISIR PSF and we applied a PSF subtraction in analogy to the image analysis of Sect. 4.4. The observed resolved emission component is not reproduced by the model. A likely explanation for this is the photoluminescence of particles with sizes between 

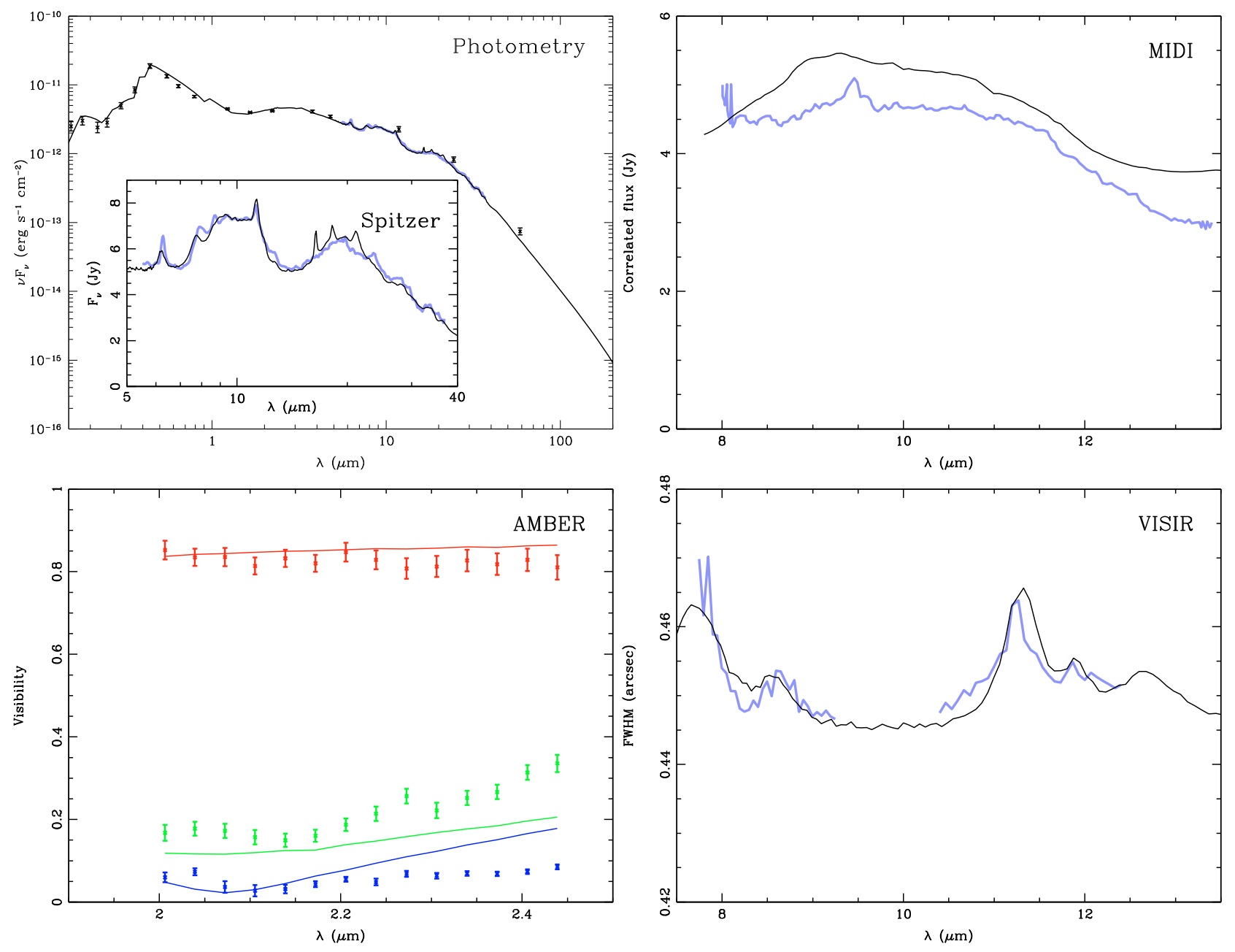

Fig. 7. The best model fit to the observables of HD 95881. The upper left panel shows the fit to the spectral energy distribution with an inset for the Spitzer spectrum. The blue line is the observed spectrum, the black line the model spectrum and the points with error bars are photometric measurements obtained from the literature. The upper right panel shows the correlated flux as obtained by MIDI. The blue line is the observed correlated flux and the black line the model. The bottom left panel shows the visibilities obtained by AMBER. The colored lines give the model results (color coding the same as Fig. 1). The bottom right panel shows in blue the VISIR FWHM as a function of wavelength and in black the model.

dust grains and PAH molecules, the so called very small grains, which are not included in our model (see Flagey et al. 2006).

\subsection{The distribution of gas and dust}

Our modeling reveals that the spatial distribution of the gas and the dust in the disk around HD 95881 is different. The dust in the upper layers of the outer disk is heavily depleted, while the gas still has a large scale height. To reproduce the near IR flux we needed to assume a scale height, which is much larger than would be obtained from vertical hydrostatic equilibrium $(\Psi=$ 2.75). We do not have an explanation for this, but we note that the value of $\Psi$ is sensitive to the assumptions on the structure and composition of the material in the inner disk (see e.g. Isella et al. 2008; Tannirkulam et al. 2008a). Furthermore, our value is well within the range of scaling parameters (1.0-3.0) needed by Acke et al. (2009) to explain the near IR SED of about half of their sample of $\sim 30$ Herbig Ae/Be stars.

The spatial distribution of the visible grain component was well constrained by the SED. The large grains could not be constrained directly, but are probably abundantly present in a settled outer disk. Observations of the millimeter flux will help to constrain the mass in this component as well as the size distribution.
We can however make an estimate of the total dust mass, based on the modeled total PAH mass $\left(5 \times 10^{-9} M_{\odot}\right)$ and the modeled PAH abundance at the inner edge $(0.25 \%)$. Assuming that at the inner rim all the dust is visible and assuming that the PAH to dust ratio is homogeneous throughout the disk we find that the total dust mass adds up to $2 \times 10^{-6} M_{\odot}$. This means that most of the dust mass resides in large grains (200:1). Using the canonical gas to dust ratio of 100 the total disk mass becomes $2 \times 10^{-4} M_{\odot}$. Note, that if the outer disk has a lower value of $\Psi$, a larger gas mass could be required to reproduce the observations.

The results presented above naturally lead to the picture of a disk in which the dust grains in the outer disk are coagulated and settled towards the midplane, while the gas is still available to keep the PAH molecules in the higher atmosphere of the disk. As already noted in the theoretical study by Dullemond et al. (2007), growth and settling of the dust grains leads to a natural increase of the relative strength of the PAH signature, as is observed in this disk and confirmed by our modeling effort.

\subsection{Context}

In general the study of Dullemond et al. (2007) showed that the natural outcome of a group I source after grain-growth and 
sedimentation of the dust is a group II source, that maintains the flaring structure for the gas. However, observational studies have shown that most group II sources lack a flaring gas distribution. Meeus et al. (2001), Acke et al. (2004, 2010) showed that group I sources display significantly more PAH emission. Acke et al. (2005) showed that group I sources have in general stronger [O I] emission. Apparently the gas of most group II sources has either dramatically decreased its scale height because of the lack of heating or the gas has been dispersed from the disk. How disks can lose their gas is currently being debated (see Hillenbrand 2008), but photoevaporation seems to be the most likely mechanism.

On the other hand there is a fair number of group II sources that do show indications of a flaring gas distribution. Some group II sources display PAH emission in their $10 \mu \mathrm{m}$ spectra, for example: HK Ori (van Boekel et al. 2005) and HD 142666 (Verhoeff et al. 2010). Some group II sources display the [O I] $6300 \AA$ line in emission, for example: HD 98922 (Acke et al. 2005). And some show both PAH and [OI] emission, for example: HD 101412 (Fedele et al. 2008). These sources all seem to be in a transitional phase from a gas rich flaring disk to a gas poor self-shadowed disk. Roughly half of the known group II sources are in this phase, which means we can infer that half of the life time of the disk of a group II source is spent on the dispersal of the gas. An estimate for this time scale is the photoevaporation time scale, which is on the order of $\sim 10^{6} \mathrm{yr}$ (Gorti \& Hollenbach 2009)

\section{Conclusions}

A comprehensive study was performed to map the distribution of the gas and dust in the protoplanetary disk around HD 95881. In the right panel of Fig. 6 we displayed a schematic representation of the disk, which puts all results in perspective. The AMBER $K$-band interferometry showed that there is an extended hot inner region with emission coming from well within the sublimation radius. The detection of the [O I] $6300 \AA$ indicated that the disk has a flaring gas surface at large distances (from one to tens of $\mathrm{AU}$ ) from the star. The finding of PAH features in the Spitzer and VISIR spectra confirmed the presence of an illuminated gas surface. The resolved VISIR spectrum traced this surface up to radii of $\sim 100 \mathrm{AU}$.

We used the radiative transfer code MCMax (Min et al. 2009) to create a model of the disk's density and temperature structure. Our model satisfactorily reproduced all of our observations. The main conclusions that followed from our model are that the inner disk contains most of the visible grains and has a puffed up inner rim, the dust grains in the outer disk have coagulated and settled towards the midplane, while the gas and PAH mixture maintain a flaring geometry. Theory predicted the existence of these type of disks (Dullemond et al. 2007), while observational trends showed that most of the sources with selfshadowed dust distributions have dispersed the bulk of their gas. In this light HD 95881 is a special source: it is in the transition phase from a gas rich flaring dust disk to a gas poor selfshadowed disk.
Acknowledgements. This research was sponsored by NWO under grant number 614.000.411. M. Min acknowledges financial support from the Netherlands Organization for Scientific Research (NWO) through a Veni grant. E. Pantin acknowledges financial support from the Agence Nationale de la Recherche (ANR) of France through contract ANR-07-BLAN-0221.

\section{References}

Acke, B., \& van den Ancker, M. E. 2004, A\&A, 426, 151

Acke, B., van den Ancker, M. E., Dullemond, C. P., van Boekel, R., \& Waters, L. B. F. M. 2004, A\&A, 422, 621

Acke, B., van den Ancker, M. E., \& Dullemond, C. P. 2005, A\&A, 436, 209

Acke, B., Verhoelst, T., van den Ancker, M. E., et al. 2008, A\&A, 485, 209

Acke, B., Min, M., van den Ancker, M. E., et al. 2009, A\&A, 502, L17

Acke, B., Bouwman, J., Juhász, A., et al. 2010, ApJ, accepted [arXiv: 1006.1130 ]

Benisty, M., Natta, A., Isella, A., et al. 2010, A\&A, 511, A74

de Zeeuw, P. T., Hoogerwerf, R., de Bruijne, J. H. J., Brown, A. G. A., \& Blaauw, A. $1999, \mathrm{AJ}, 117,354$

Draine, B. T., \& Li, A. 2001, ApJ, 551, 807

Draine, B. T., \& Li, A. 2007, ApJ, 657, 810

Dullemond, C. P., \& Dominik, C. 2004, A\&A, 417, 159

Dullemond, C. P., Dominik, C., \& Natta, A. 2001, ApJ, 560, 957

Dullemond, C. P., Apai, D., \& Walch, S. 2006, ApJ, 640, L67

Dullemond, C. P., Henning, T., Visser, R., et al. 2007, A\&A, 473, 457

Eisner, J. A., Lane, B. F., Hillenbrand, L. A., Akeson, R. L., \& Sargent, A. I. 2004, ApJ, 613, 1049

Eisner, J. A., Chiang, E. I., Lane, B. F., \& Akeson, R. L. 2007, ApJ, 657, 347

Fedele, D., van den Ancker, M. E., Acke, B., et al. 2008, A\&A, 491, 809

Flagey, N., Boulanger, F., Verstraete, L., et al. 2006, A\&A, 453, 969

Geers, V. C., van Dishoeck, E. F., Visser, R., et al. 2007, A\&A, 476, 279

Gorti, U., \& Hollenbach, D. 2009, ApJ, 690, 1539

Guhathakurta, P., \& Draine, B. T. 1989, ApJ, 345, 230

Hillenbrand, L. A. 2008, Physica Scripta Volume T, 130, 014024

Houk, N., \& Cowley, A. P. 1975, Michigan Catalogue of two-dimensional

spectral types for the HD star (Ann Arbor: University of Michigan, Departement of Astronomy)

Hughes, A. M., Wilner, D. J., Qi, C., \& Hogerheijde, M. R. 2008, ApJ, 678, 1119 Isella, A., Tatulli, E., Natta, A., \& Testi, L. 2008, A\&A, 483, L13 Isella, A., Carpenter, J. M., \& Sargent, A. I. 2009, ApJ, 701, 260

Juhász, A., Bouwman, J., \& Henning, T. 2010, ApJ, submitted

Kraus, S., Preibisch, T., \& Ohnaka, K. 2008, ApJ, 676, 490

Lagage, P. O., Pel, J. W., Authier, M., et al. 2004, The Messenger, 117, 12

Lagage, P.-O., Doucet, C., Pantin, E., et al. 2006, Science, 314, 621

Leinert, C., Graser, U., Waters, L. B. F. M., et al. 2003, in Interferometry for Optical Astronomy II, ed. W. A. Traub, Proc. SPIE, 4838, 893

Leinert, C., van Boekel, R., Waters, L. B. F. M., et al. 2004, A\&A, 423, 537

Meeus, G., Waters, L. B. F. M., Bouwman, J., et al. 2001, A\&A, 365, 476

Meynet, G., Maeder, A., Schaller, G., Schaerer, D., \& Charbonnel, C. 1994, A\&AS, 103, 97

Min, M., Hovenier, J. W., \& de Koter, A. 2005, A\&A, 432, 909

Min, M., Dullemond, C. P., Dominik, C., de Koter, A., \& Hovenier, J. W. 2009, A\&A, 497, 155

Pantin, E., Doucet, C., Käufl, H. U., et al. 2008, in SPIE Conf. Ser., 7014, 701424

Pantin, E., Siebenmorgen, R., Käufl, H. U., \& Sterzik, M. 2009, in Science with the VLT in the ELT Era, ed. A. Moorwood, Ap\&SS Proc. (Springer), 261

Preibisch, T., Ossenkopf, V., Yorke, H. W., \& Henning, T. 1993, A\&A, 279, 577 Siebenmorgen, R., Kruegel, E., \& Mathis, J. S. 1992, A\&A, 266, 501

Tannirkulam, A., Monnier, J. D., Harries, T. J., et al. 2008a, ApJ, 689, 513

Tannirkulam, A., Monnier, J. D., Millan-Gabet, R., et al. 2008b, ApJ, 677, L51 Tatulli, E., Millour, F., Chelli, A., et al. 2007, A\&A, 464, 29

van Boekel, R., Waters, L. B. F. M., Dominik, C., et al. 2004, A\&A, 418, 177

van Boekel, R., Min, M., Waters, L. B. F. M., et al. 2005, A\&A, 437, 189

van der Plas, G., van den Ancker, M. E., Fedele, D., et al. 2008, A\&A, 485, 487

Verhoeff, A. P., Waters, L. B. F. M., Veerman, H., et al. 2010, A\&A, submitted 